\title{
Hot working behaviour and processing maps of duplex cast steel
}

\begin{abstract}
In this paper the hot working behaviour of medium carbon duplex cast steel is studied using uniaxial hot compression tests over a temperature range varying from $700^{\circ} \mathrm{C}$ to $1000^{\circ} \mathrm{C}$ and at different strain rates ranging from $10^{-4}$ to $10^{-1} \mathrm{~s}^{-1}$. A model based on a variant of a dynamic materials model was employed to construct processing maps. These maps delineate the safe and unsafe domains. The safe domains, associated with dynamic recrystallization and dynamic recovery, can be chosen to optimize the hot workability of the studied material. Whereas, the unsafe domain is to be avoided because it is associated with plastic deformation instabilities. The domain associated with dynamic recrystallization is centred at $1000^{\circ} \mathrm{C}$ and $10^{-4} \mathrm{~s}^{-1}$ with a peak energy dissipation efficiency of about $40 \%$, while the domain associated with dynamic recovery is centred at $700^{\circ} \mathrm{C}$ and $10^{-4} \mathrm{~s}^{-1}$ with a peak energy dissipation efficiency of about $27 \%$. The unsafe hot working domain, spread over the entire temperature range and moderate to high strain rates, predicts the appearance of flow instabilities, in the form of shear bands and intergranular cracks. To validate the obtained results, microstructural observations corresponding to different processing conditions are presented.
\end{abstract}

Keywords: Compression test; Dynamic recrystallization; Flow instability; Hot deformation; Processing maps

\section{Introduction}

The globalization of markets combined with an increasing number of product variants poses a serious challenge to the mechanical manufacturing companies as they are now forced to compete not only in their own country, but they face international competition. After all, what prevails is not only increasing productivity but also the ability to adapt to a new quality of flexible behaviour. Therefore, the need for greater competitiveness, lower costs and increasing productivity require all areas of technology to be very demanding in terms of optimization of forming processes in order to obtain reliable parts with precise dimensions and a longer service life. As is well known, hot forming is distinguished from other fabrication techniques (casting, machining, welding, etc.) by introducing large degrees of strain that cause specified microstructural changes. These changes affect the mechanical properties of the deformed material such as the flow stress. So, to optimize the mechanical parts design during a hot forming process it is essential to carry out an adequate characterization of material flow behavior [1].

Duplex cast steels (DCS) are a family of steels characterized by a microstructure containing two phases: ferrite $(\alpha)$ and austenite $(\gamma)$. The applications of this material are steadily increasing in importance, especially for components working in corrosive and erosive environments $[2,3]$. It offers a combination of the advantages of both ferritic and austenitic steels. In the last few years, this 
kind of steel has been widely used in the nuclear and chemical industries [4]. However, its use is still limited due to its limited formability and there is a growing interest in improving the understanding of the physical and mechanical phenomena that affect its workability in order to expand its field of applications. The hot working of the DCS requires special attention; it is well known that austenite and ferrite have a dissimilar flow behaviour during hot working. When they are deformed jointly the deformation is not distributed uniformly, so the workability of the deformed steel can decrease dramatically, causing the formation of microstructural defects (cracks, cavities, flow localization, shear bands, surface cracks, etc.) [5-7]. In order to establish the optimum hot working processing conditions, modelling workability, influenced by process parameters such as applied temperature, strain rate and strain, become very important.

Various models and approaches have been used to characterize the hot working processes $[8-10]$ and one of the most important models is the dynamic materials model (DMM) $[8,10-12]$. This model has been found to be a very valuable tool for analysing and optimizing the hot workability of a wide range of materials. But, regrettably, DMM is still not being recognized by several hot working researchers because of the anomalies that they have encountered on its physical and conceptual foundations [9, 13-16]. In DMM, to describe the behaviour of the deformed material, Prasad et al. used a power constitutive law $\left(\sigma=K \dot{\varepsilon}^{m}\right)$ to calculate the energy dissipation efficiency and the instability parameter. Narayana Murthy et al. showed that employing a power constitutive law, indiscriminately for higher and lower domains of stress, is erroneous because it is well known that in the forming processes the power constitutive law has only been used for the analysis of steady state flow stress data under low stress conditions. Therefore, an alternative approach has been proposed and it can be considered as a variant of the dynamic materials model (VDMM) [17-20].

Although various research work has been carried out to study the hot working of different kinds of steels using DMM, at present there is little work on the workability of DCS during hot forming. The main objective of the present work is to characterize the hot working behaviour of a medium carbon DCS using processing maps constructed on the basis of DMM and its variant, VDMM. From analysis of these maps, optimized parameters of stable hot workability were determined and unstable parameter domains were validated by the microstructural observations. Furthermore, a comparative study of the obtained results has been made to show the difference between the positions of stability domains predicted by the two models.

\section{Workability and DMM flow stability criteria}

In industrial conditions, hot forming processes involve complex deformation patterns and generate extreme deformations of the initial geometry. The development of possible plastic instabilities can compromise the progress of these processes causing the onset of rapid crack propagation before the final rupture of the hot formed piece. In the attempt to optimize process control parameters, many researchers have proposed several criteria capable of predicting the onset of plastic instabilities [8, 9, 21-23]. These criteria are based on the simultaneous intervention, during the hot deformation, of numerous metallurgical mechanisms such as strain hardening, dynamic recovery (DRV), dynamic recrystallization (DRX), etc.

In the dynamic materials model the hot worked piece does not store energy but dissipates it as microstructural changes and a temperature rise [8]. Following this consideration, Prasad demonstrated that the energy dissipation efficiency $\left(\eta_{\mathrm{P}}\right)$ may be expressed as a dimensionless parameter:

$\eta_{\mathrm{P}}(\dot{\varepsilon}, T)=\frac{2 m}{m+1}$

where $m$ is the strain rate sensitivity parameter of flow stress.

The variation of $\eta_{\mathrm{P}}(\dot{\varepsilon}, T)$ with temperature and strain rate constitutes the energy dissipation efficiency map, which exhibits various domains that may be correlated with "safe" and "unsafe" mechanisms that occur at different strain rates and temperature combinations. It is necessary to point out that optimum hot working conditions are attained when the energy dissipation efficiency is maximum.

Moreover, Prasad established an analytical expression for the condition of the onset of the flow instability, based on the extremum principles of irreversible thermodynamics applied to large plastic flow, and is as follows:

$\xi_{\mathrm{P}}(\dot{\varepsilon}, \mathrm{T})=\frac{\partial \log [m / m+1]}{\partial \log \dot{\varepsilon}}+m<0$

The variation of the instability parameter $\xi_{\mathrm{P}}(\dot{\varepsilon}, T)$ with temperature and strain rate constitute the flow instability map. This map indicates the regions where the instability parameter is negative, and therefore delimits the zones to avoid during hot forming.

As can be seen from above, the strain rate sensitivity parameter $m$ is the key to defining both the energy dissipation efficiency $\left(\eta_{\mathrm{P}}\right)$ and the instability parameter $\left(\xi_{\mathrm{P}}\right)$. In literature, it is well known that when a power constitutive law is employed, to describe the behaviour of materials in hot

Table 1. Chemical composition of studied steel (wt.\%), balance Fe.

\begin{tabular}{|c|c|c|c|c|c|c|c|c|c|c|c|}
\hline $\mathrm{C}$ & $\mathrm{Mn}$ & $\mathrm{Si}$ & $\mathrm{P}$ & $\mathrm{S}$ & $\mathrm{Ni}$ & $\mathrm{Al}$ & $\mathrm{Mo}$ & $\mathrm{Nb}$ & $\mathrm{W}$ & $\mathrm{N}$ & $\mathrm{Cr}$ \\
\hline 0.31 & 0.79 & 1.07 & 0.03 & 0.32 & 0.083 & 0.018 & 0.49 & 1.28 & 0.08 & 0.34 & 26.7 \\
\hline
\end{tabular}


forming processes, the strain rate sensitivity parameter $m$ becomes a parameter totally independent of strain rate $[9$, $14,15,24,25]$. Therefore, the instability condition established by Prasad et al., in Eq. (2), reduces to simply $m<0$ for an eventual metallurgical instability. This assumption is considered by several authors to be a scientific inconsistency, because the experimental values of the parameter $m$ should be between zero and one [14-20]. To overcome this inconsistency, Narayana Murthy et al. [9, 17-20] presented a variant of the dynamic materials model (VDMM) as a new methodology to calculate the energy dissipation efficiency $\eta_{\mathrm{N}}(\dot{\varepsilon}, T)$ and established a new flow instability parameter $\xi_{\mathrm{N}}(\dot{\varepsilon}, T)$, applicable for any type of flow curve. The detailed procedures to develop the VDMM are described in previous papers, where the energy dissipation efficiency $\eta_{\mathrm{N}}$ $(\dot{\varepsilon}, T)$ is given by:

$\eta_{\mathrm{N}}(\dot{\varepsilon}, \mathrm{T})=\frac{1}{2}\left[1-\frac{1}{\sigma \dot{\varepsilon}}\left(\left(\frac{\sigma \dot{\varepsilon}}{m+1}\right)_{\dot{\varepsilon}=\dot{\varepsilon}_{\min }}+\int_{\dot{\varepsilon}_{\min }}^{\dot{\varepsilon}} \sigma \mathrm{d} \dot{\varepsilon}\right)\right]$

$\dot{\varepsilon}_{\text {min }}$ represents the lowest strain rate used in the hot compression tests.

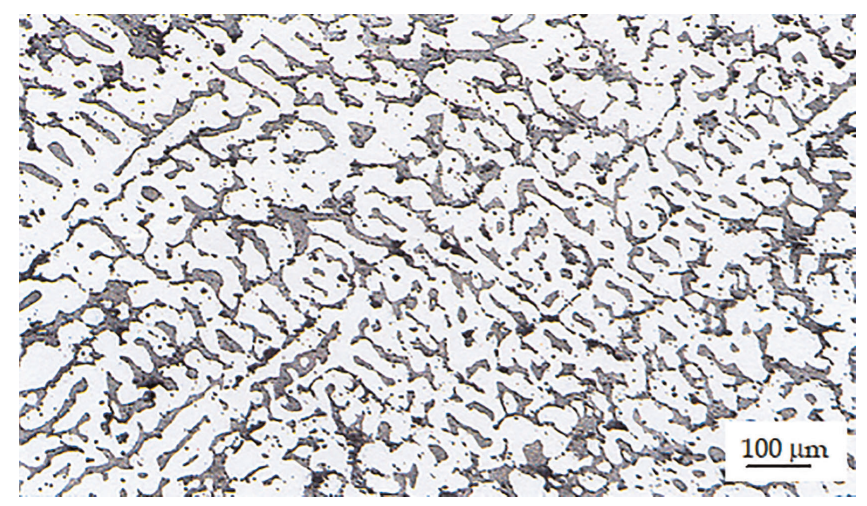

Fig. 1. Initial microstructure of the studied steel.

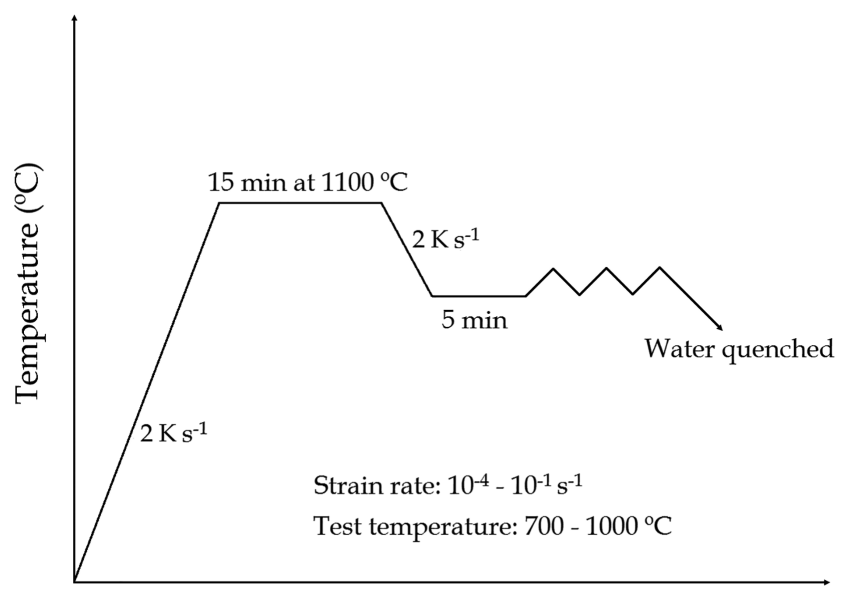

Time (s)

Fig. 2. Schematic illustration of hot compression test.
Moreover, the new VDMM condition for the flow instability is:

$\xi_{\mathrm{N}}(\dot{\varepsilon}, T)=\frac{2 m}{\eta_{\mathrm{N}}}-1<0$

\section{Experimental procedure}

The studied steel was melted in a medium frequency induction furnace and produced by continuous casting at $1620^{\circ} \mathrm{C}$. The chemical composition of the steel, received in the form of keel blocks, is shown in Table 1. The typical initial microstructure of the cast steel is shown in Fig. 1 and it was analysed using automatic image analysis software. The volume fractions of ferrite and austenite have been estimated as $\sim 20 \%$ and $\sim 80 \%$, respectively. In this kind of steel, the presence of the ferrite in the structure is desirable to improve its weldability and its resistance to stress corrosion cracking.

To characterize the hot flow behaviour of the studied steel, uniaxial hot compression tests were performed, at constant true strain rate, on an INSTRON 4507 electromechanical machine with a $100 \mathrm{kN}$ load cell and equipped with an electrical resistance furnace, that ensured a constant and homogeneous temperature, and computer programs that allow obtaining directly the true stress-true strain curve at end of each test. The compression tests were carried out in the temperature range of 700$1000{ }^{\circ} \mathrm{C}$ with $50{ }^{\circ} \mathrm{C}$ intervals and at different strain rates varying from $10^{-4}$ to $10^{-1} \mathrm{~s}^{-1}$. The dimensions of the compression cylindrical samples were $11.4 \mathrm{~mm}$ in length and $7.6 \mathrm{~mm}$ in diameter. To reduce the friction effects, boron nitride lubricant was deposited on both contacting surfaces of specimens.

Prior to testing, each compression test sample was soaked at $1100^{\circ} \mathrm{C}$ for $15 \mathrm{~min}$ in an argon atmosphere followed by cooling at $2 \mathrm{~K} \mathrm{~s}^{-1}$ to the test temperature and maintained there for $5 \mathrm{~min}$. before starting the compres-

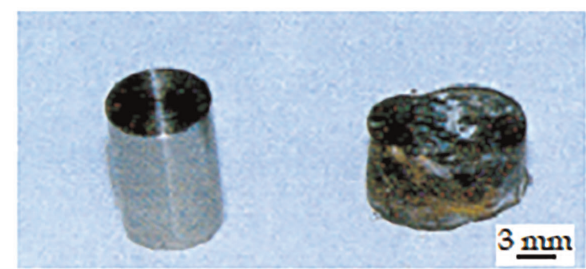

(a)

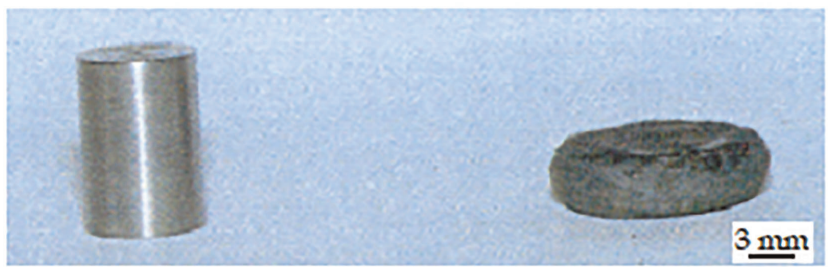

(b)

Fig. 3. Macro-morphologies of selected specimens deformed at: (a) $T=750{ }^{\circ} \mathrm{C}$ and $\dot{\varepsilon}=0.1 \mathrm{~s}^{-1}$, and (b) $T=950^{\circ} \mathrm{C}$ and $\dot{\varepsilon}=10^{-4} \mathrm{~s}^{-1}$.

Int. J. Mater. Res. 112 (2021) 7 
sion test. Figure 2 shows schematic illustration of the hot compression test. The experimental flow curves were obtained immediately after each compression test. The deformed samples were water cooled immediately after testing to avoid changes in the deformed microstructure. Specimens for metallographic examination were sectioned parallel to the compression axis and prepared by grinding and polishing, and then etched using a solution of $\left(\mathrm{HCL}+\mathrm{HNO}_{3}+\mathrm{H}_{2} \mathrm{O}\right)$. A metallographic study was performed using automatic image analysis techniques to determine the volume percent of the two phases in the starting specimens and to follow microstructural changes through hot deformation.

Figure 3 shows macro-morphologies of selected specimens deformed in hot compression conditions. In specimens deformed at low temperatures and high strain rates (Fig. 3a) surface cracking was found to occur, suggesting that material exhibits low ductility at these temperatures and probably onset of damage mechanisms. By contrast, at high temperatures and low strain rates the material flow is quite acceptable (Fig. 3b).

\section{Results and discussion}

Figure 4 shows the flow curves of the steel obtained at various temperatures and strain rates. The different characteristics of true stress-true strain curves can be explained bearing in mind the coexistence of ferrite and austenite in the microstructure of DCS and that the dominant softening mechanisms are DRV and DRX. It is well known that the hot deformation behaviour of ferrite and austenite is different; normally ferrite is restored by DRV and austenite is restored by DRX [5, 26-28]. In the flow curves, when the compression test starts, the flow stress increases rapidly to attain a peak stress value and subsequently decreases or it remains constant when the strain is increased. It is generally recognized that the presence of the peak stress in the flow curves indicates the existence of DRX. Also, in the curves in which there is no well-defined peak stress but is steady state, the dominant restoration mechanism is DRV. In these curves, the flow behaviour is a result of dynamic equilibrium reached between generation and elimination of dislocations. Fig-
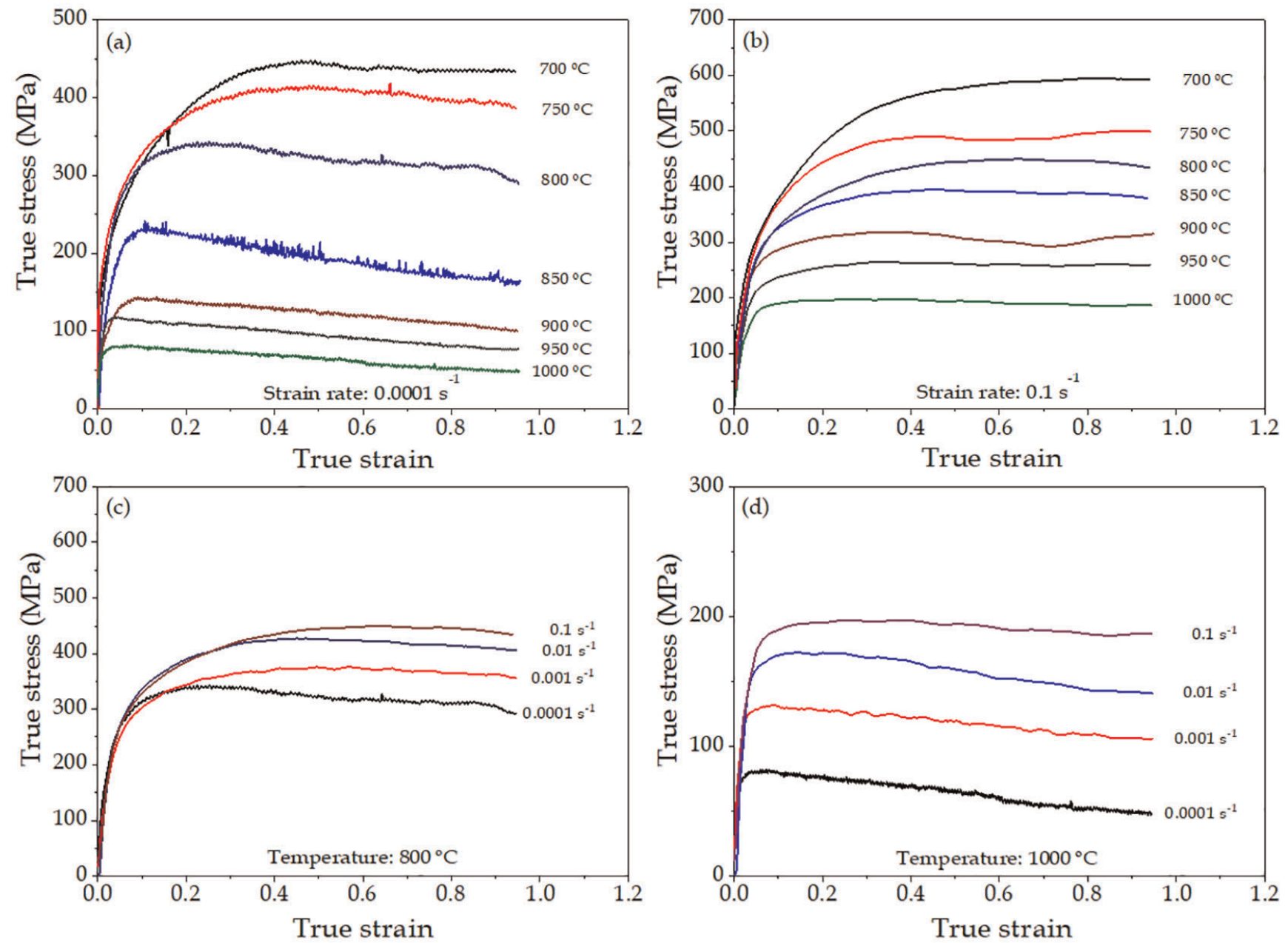

Fig. 4. Flow curves obtained at: (a) strain rate of $10^{-4} \mathrm{~s}^{-1}$ and various temperatures, (b) strain rate of $0.1 \mathrm{~s}^{-1}$ and various temperatures, (c) temperature of $800{ }^{\circ} \mathrm{C}$ and various strain rates, and (d) temperature of $1000{ }^{\circ} \mathrm{C}$ and various strain rates. 
ure 5 shows the variation of peak stress and steady state stress with temperature and strain rate. As expected, temperature and strain rate have important effects on the flow behaviour of the studied steel, peak stresses and steady state stresses increase when the strain rate increases or the deformation temperature decreases. The effect of temperature can be easily interpreted considering that at temperatures where the diffusion mechanism is slow, flow behaviour become increasingly difficult producing higher values of flow stresses [29]. The increase in the peak stresses and steady state stresses with strain rate can be attributed to the increase in the rate of the strain hardening mechanism and decrease in the restoration mechanisms (DRV and DRX). Moreover, it is well known that higher strain rates produce higher dislocation density and consequently higher strain hardening due to dislocation interactions. To characterize the flow softening caused by DRX degree of softening $X$, defined in Eq. (5), has been used.
A contour map representing iso- $X$ variations with temperature and strain rate is shown in Fig. 6.

$X=100 \frac{\sigma_{\mathrm{P}}-\sigma_{\mathrm{SS}}}{\sigma_{\mathrm{P}}}$

where $\sigma_{\mathrm{P}}$ and $\sigma_{\mathrm{SS}}$ are peak and steady state flow stress, respectively.

In the softening degree contour map, it is noticeable that the flow softening behaviour of the studied steel is sensitive to deformation temperature and strain rate. At low strain rates and high temperatures domain, the maximum softening degree is reached at $1000^{\circ} \mathrm{C}$ and $10^{-4} \mathrm{~s}^{-1}$. As seen above, in this domain the flow softening is mainly induced by the DRX. However, with the increase of strain rate or decrease of temperature, the degree of softening becomes low. In this case, the softening flow is mainly caused by the DRV.

The processing maps constructed on the basis of DMM and VDMM at a strain of 0.6 are shown in Fig. 7. Although
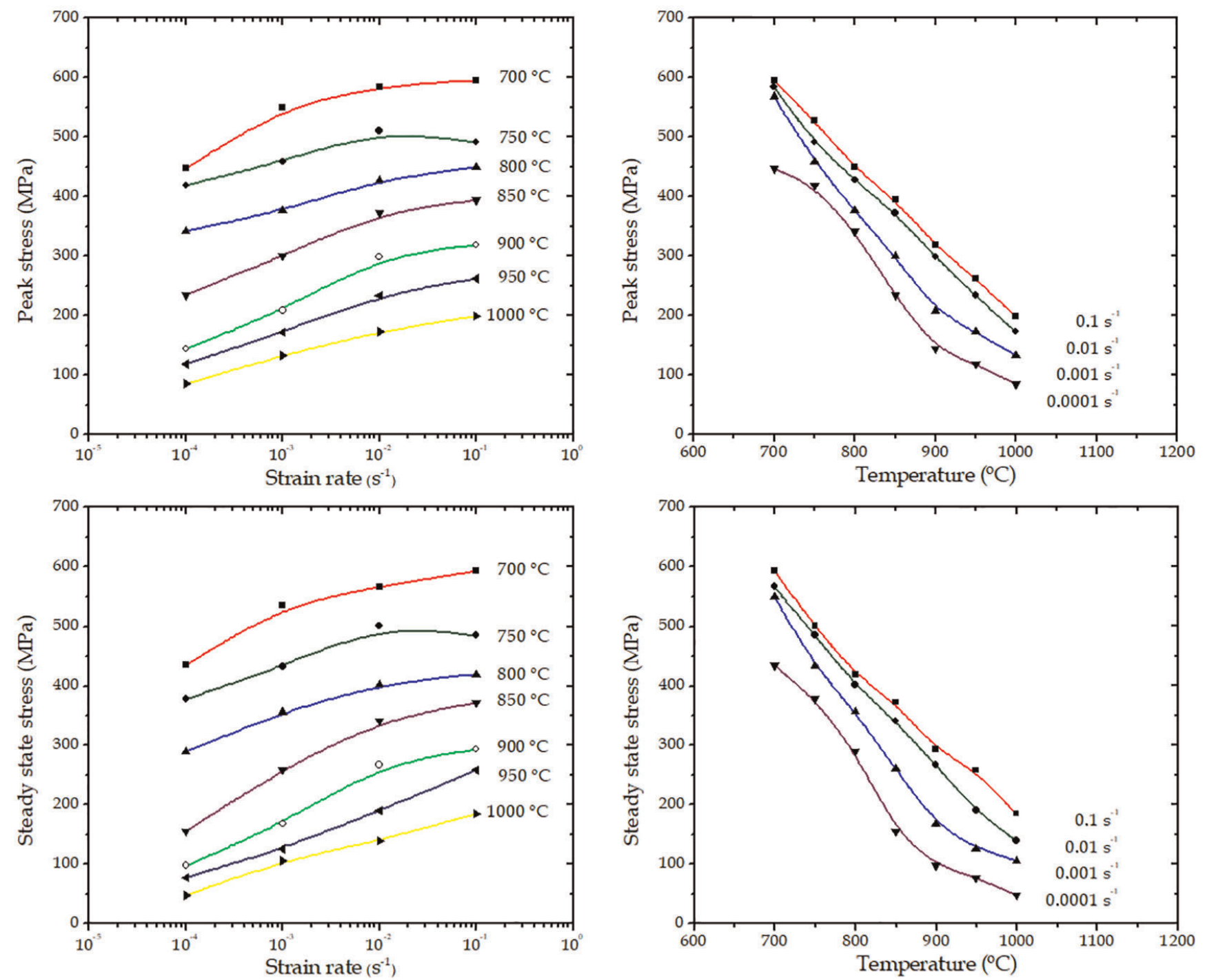

Fig. 5. Variation of peak stress and steady state stress with temperature and strain rate. 
the numerical integration procedure used in the VDMM, to compute the energy dissipation efficiency, is different to that used in the DMM, the values of energy dissipation efficiency defined in DMM and VDMM (Fig. 7a and b) are similar. This similitude suggest that the constitutive behaviour of the studied steel obeys a power law. Nevertheless, the instability maps (Fig. 7c and d) reveal the existence of some differences concerning the instability parameter values and position of domains situated at the upper part of the maps.

In Fig. 7a and $b$ the energy dissipation maps exhibit three significant domains; the first is centred at $1000^{\circ} \mathrm{C}$ and $10^{-4}$ $\mathrm{s}^{-1}$ and with a peak energy dissipation efficiency of about $40 \%$, the second is centred at $700^{\circ} \mathrm{C}$ and $10^{-4} \mathrm{~s}^{-1}$ with a peak energy dissipation efficiency of about $27 \%$ The third domain appears in the upper left of the map with the lowest energy dissipation efficiency of about $2 \%$ (Fig. 7a and b).

In literature, it is well accepted that the DRX domain is characterized by energy dissipation efficiency values of about $30-50 \%$ while the energy dissipation efficiency values of the DRV domain are about $30 \%[30,31]$. Hence, the first domain

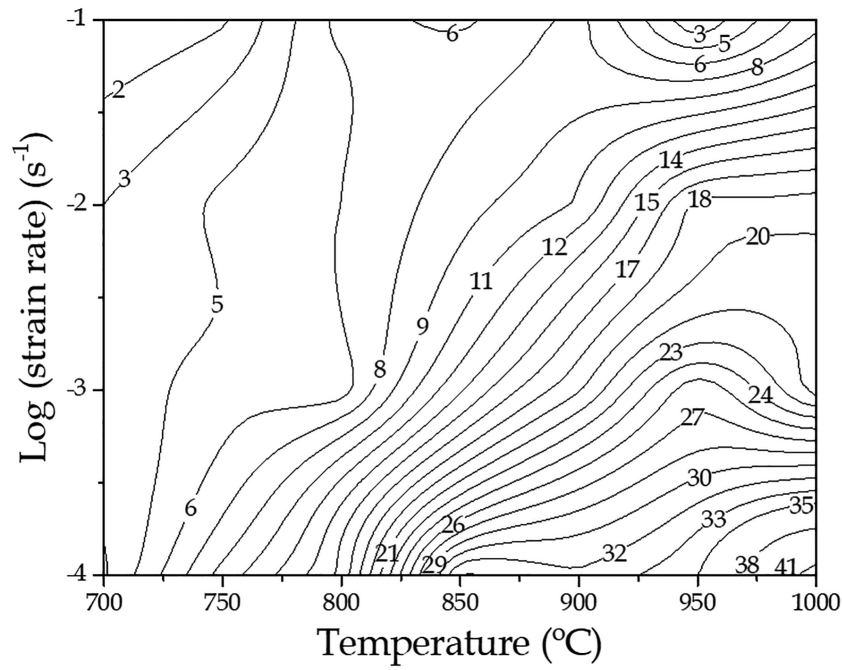

Fig. 6. Contour map representing degree of softening $X$ of the steel. (a)

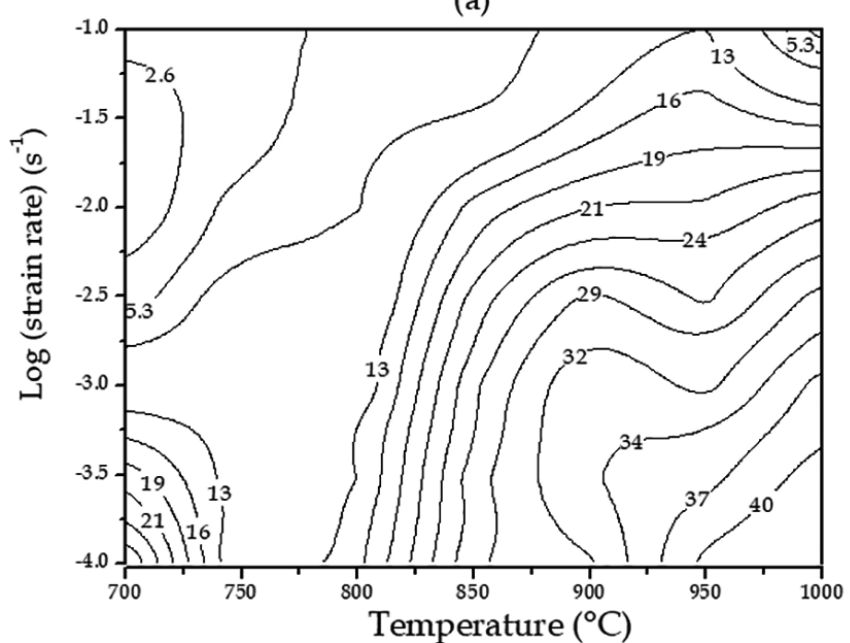

(c)

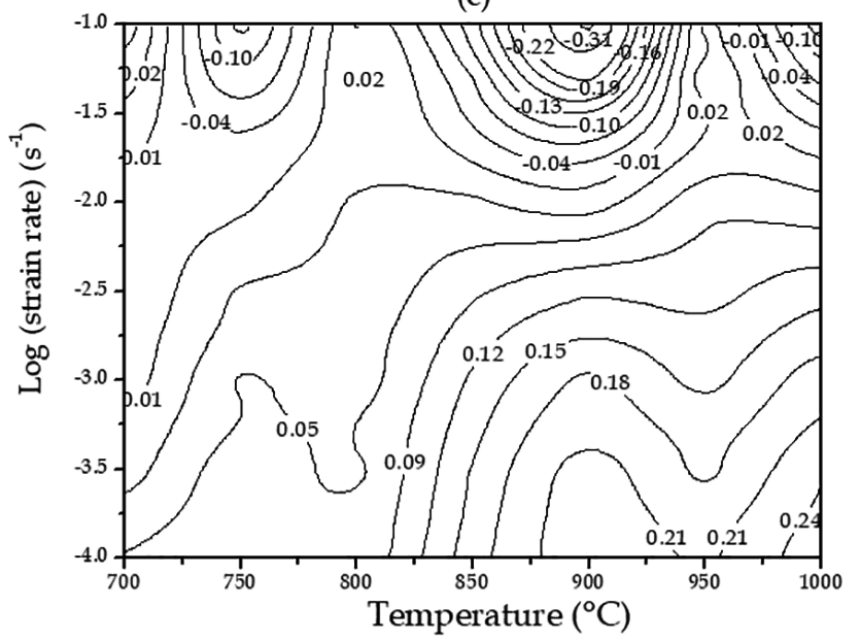

(b)

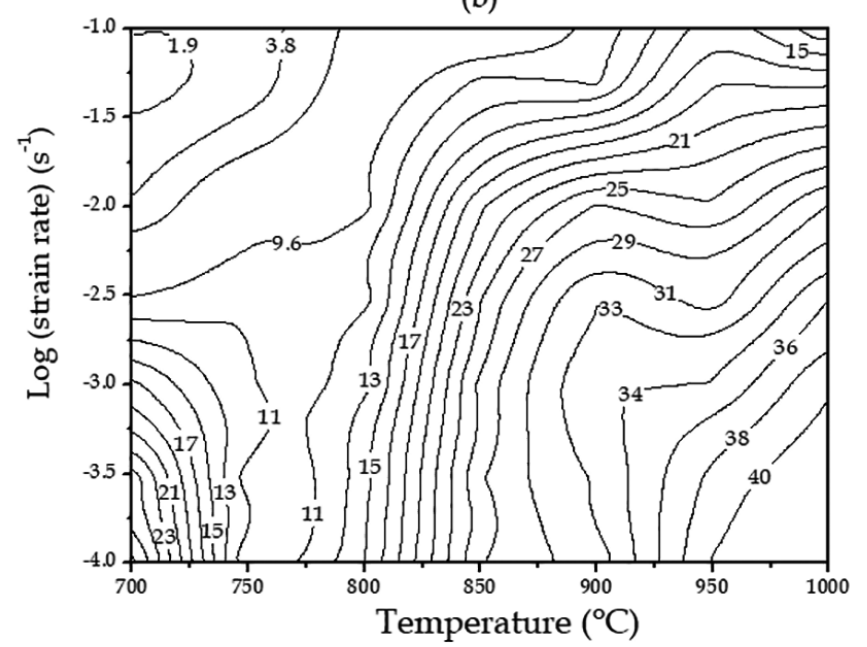

(d)

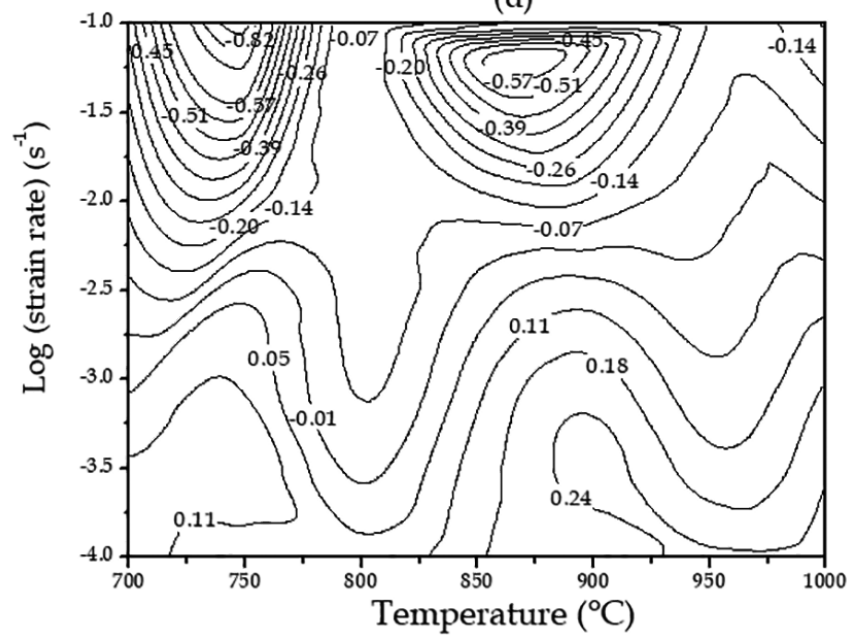

Fig. 7. Processing maps of the studies steel at a strain of 0.6: (a) DMM energy dissipation map, (b) VDMM energy dissipation map, (c) DMM flow instability map, (d) VDMM flow instability map. 


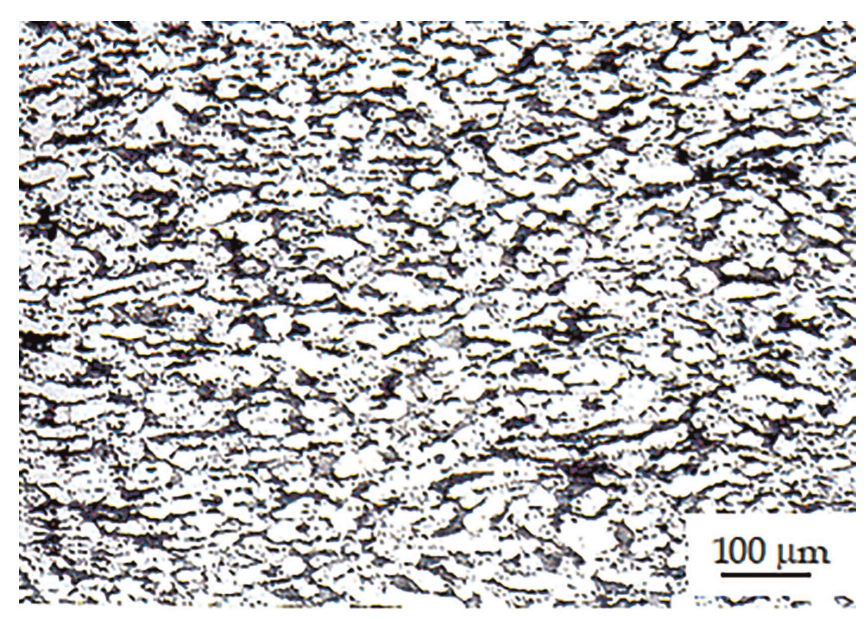

a)

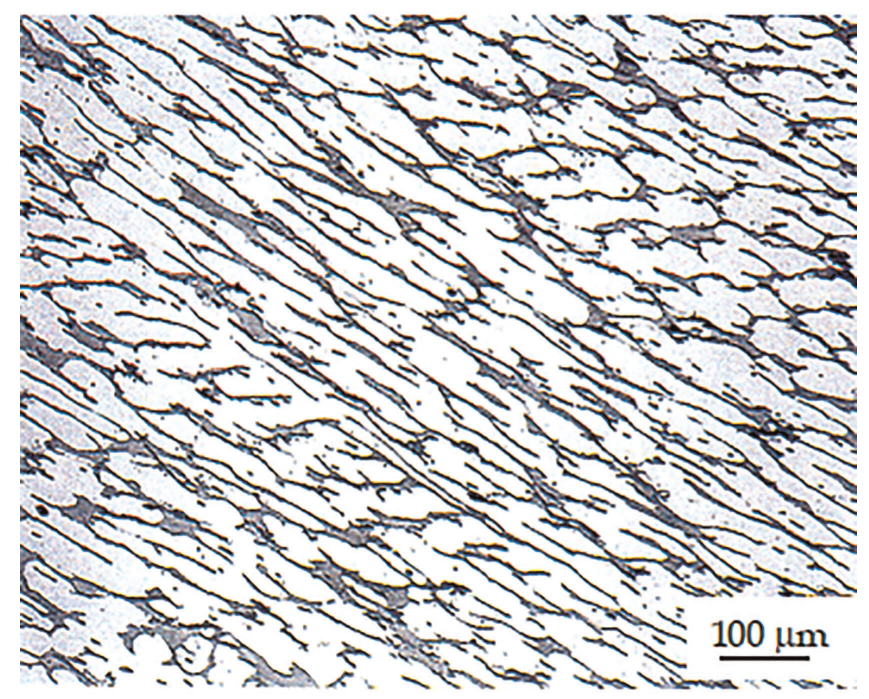

b)

Fig. 8. Microstructure of specimens deformed at: (a) $1000^{\circ} \mathrm{C}$ and $10^{-4} \mathrm{~s}^{-1}$, (b) $700^{\circ} \mathrm{C}$ and $3 \cdot 10^{-4} \mathrm{~s}^{-1}$.

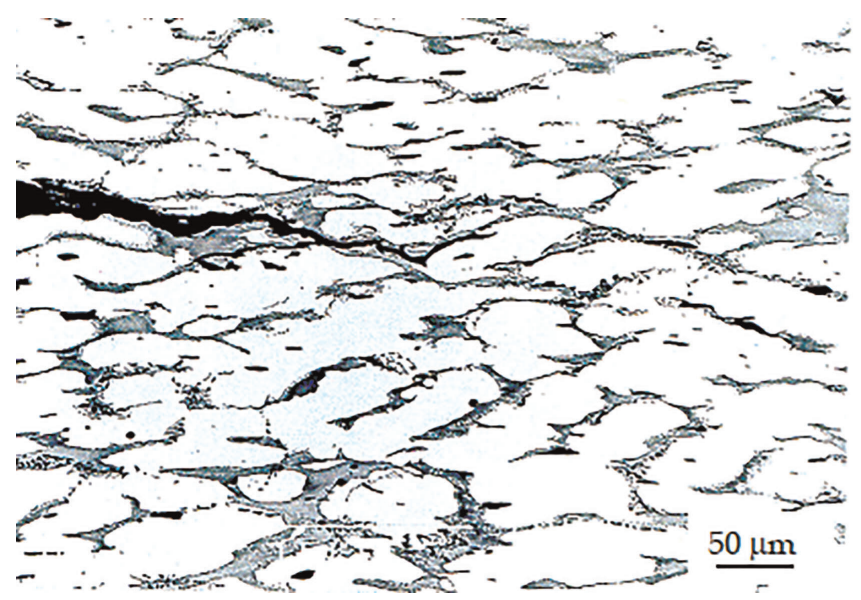

a) can be easily associated with DRX of the austenite phase, because in this domain, the flow curves (Fig. 4) show clearly continuous softening with a single peak behaviour followed by a steady state. This is emphasized by the fact, shown in Fig. 6, that degree of softening $X$ reaches its highest values in the mentioned domain. The microstructure of the specimen deformed in this domain $\left(1000^{\circ} \mathrm{C}\right.$ and $\left.10^{-4} \mathrm{~s}^{-1}\right)$ is shown in Fig. 8a. As can be observed, the microstructure shows DRX characteristics with structure considerably refined in comparison with initial one. Furthermore, and for additional confirmation, observation of Fig. 7c and d shows that the instability parameter takes its maximum positive values in this domain, and it is generally recognized that in the safe domains of hot working processes, the instability parameter is characterized by high positive values [32]. As is obvious, the domain with negative $\xi(\dot{\varepsilon}, T)$ values is nearly situated at the domain where the energy dissipation reaches its minimum values. In the second domain, the energy dissipation efficiency value is less than $30 \%$ and the flow curves show a typical behaviour of DRV (Fig. 4). For this case of low temperatures and low strain rates, DRV is expected to be the controlling softening process in the hot deformation since the $X$ values in this domain become increasingly smaller (Fig. 6). Typical microstructures of specimens deformed in this domain (Fig. 8b) exhibit a mixed of austenitic grain structure and elongated ferritic grains along the applied deformation, which is a significant characteristic of the dynamically recovered ferrite structure [26, 27]. In this micrograph, a small amount of DRX nucleis can be seen within the austenite structure. It is well accepted that ferrite and austenite behave differently during hot working processes; due to its high stacking fault energy, ferritic structure tends to soften more by DRV than by DRX, while austenite, with relatively low stacking fault energy, is normally softened by DRX [5, 26-28]. It is important to emphasize that both DRX and DRV are considered safe processes, where DRX is more efficient than DRV and is preferred for hot forming processes, since it improves in-

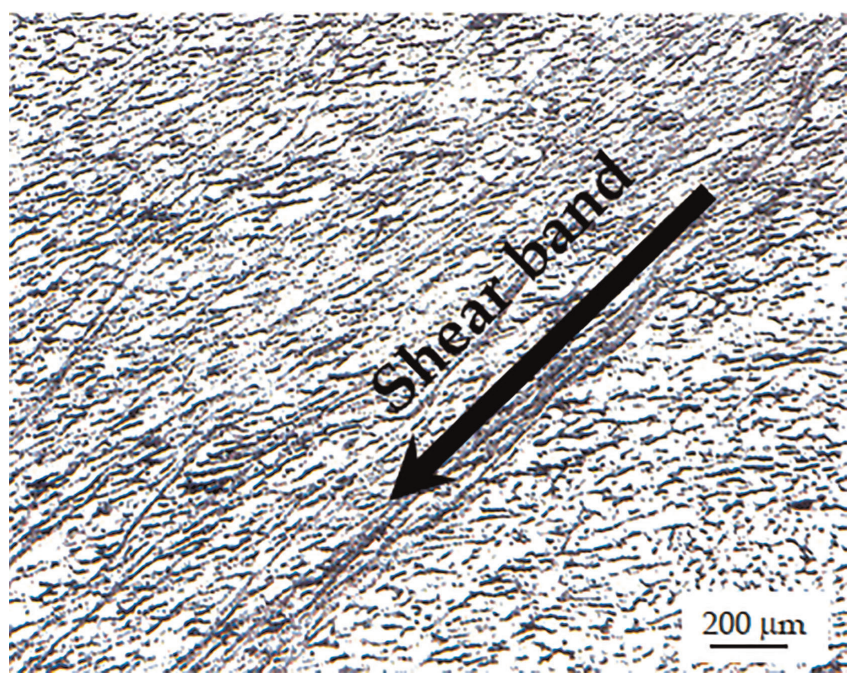

b)

Fig. 9. Microstructures of steel specimens deformed at: (a) $700{ }^{\circ} \mathrm{C}$ and $0.1 \mathrm{~s}^{-1}$, (b) $850{ }^{\circ} \mathrm{C}$ and $0.1 \mathrm{~s}^{-1}$. 
trinsic workability by reducing the flow stress and reconstituting the microstructure. Also, DRX produces more dissipation energy, through the formation of new grain structure (free of dislocation) and the migration of the boundaries, than DRV where the energy is mainly dissipated through dislocations annihilation. It is important to note that the relationship between microstructural evolution and restoration mechanisms during the hot working processes of DSS has been well documented and recognized in previous studies [5, 26-29, 33]. However, the restoration processes dominant in constituent phases and the acting dynamic softening mechanisms in DSS are still controversial. Therefore, to have a deeper understanding more research is needed, doing further microstructural and microscopic characterizations.

Despite the differences found in the contour shape and the instability parameter values, the instability maps based on the DMM and VDMM criteria (Fig. 7c and d) are similar. In these maps, the domain spread over the entire temperature range and moderate - high strain rates of the instability map show negative values of the instability parameter. As stated above, domains in which instability parameter values are negative are characterized by low energy dissipation efficiency, as depicted in Fig. 7a and b, indicating low intrinsic workability and probably the presence of some kind of flow instability. Hence, this instability domain has to be avoided during the hot forming process of the studied steel. As pointed out in several research papers $[8,11]$, the microstructural manifestations of plastic flow instabilities are various but the most common are attributed to the occurrence of adiabatic shear bands, flow localization, intergranular cracking, void formation and so forth.

The instability predictions of DMM and VDMM processing maps were validated by microstructural observations, shown in Fig. 9, and by the observation of the superficial cracks in the macro-morphologies of the specimen deformed under conditions of the unstable domain (Fig. 3a).

The microstructure of the specimen deformed at $700^{\circ} \mathrm{C}$ and $0.1 \mathrm{~s}^{-1}$ (Fig. 9a) shows the appearance and propagation of cracks in the ferrite/austenite interface and preferentially in ferrite, due to the presence of a large amount of carbides unevenly distributed in the ferrite phase. In cast duplex steels, the presence of the carbides could be the source of stress concentration areas and cracks during deformation, which produces considerable variations in its mechanical properties and reduce its toughness and plasticity [34, 35]. Also, it can be seen in Fig. $9 \mathrm{~b}$ that the microstructure of the specimen deformed at $850^{\circ}$ and $0.1 \mathrm{~s}^{-1}$ exhibit shear bands oriented at about $45^{\circ}$ with respect to the compression axis. As mentioned above, the presence in the microstructure of ferrite and austenite (which have significantly different mechanical properties) should be considered as the main reason for shear band appearance. In most literature, shear bands appear during hot working processes to facilitate the imposed deformation to continue among the ferrite and austenite phases when slip and twinning mechanisms are hampered $[33,36,37]$. Since it is considered to be a microstructure region where plastic flow is highly localized and related to uneven deformation, shear bands could be the source of flow instabilities and heterogeneous microstructures. These instability manifestations should be avoided during the hot working process of the studied steel.

\section{Conclusions}

The hot working behaviour of medium carbon duplex cast steel has been studied using Prasad's criterion (DMM) and Narayana Murthy's criterion (VDMM). The analysis of the obtained results of this study can be summarised as follows:

1. Despite the strict mathematical procedure used in the VDMM, its results are very similar to DMM's. This similitude suggests that the constitutive behaviour of the studied steel obeys a power law.

2. The studied steel undergoes single peak dynamic recrystallization in the domain centred at about at $1000^{\circ} \mathrm{C}$ and $10^{-4} \mathrm{~s}^{-1}$ and with a peak energy dissipation efficiency of about $40 \%$, which may be considered as the optimum domain for hot working.

3. The material exhibits flow instabilities, in the form of shear bands and intergranular cracks, in the domain located in the upper part of the instability map. This instability domain has to be avoided during hot forming processes of the studied steel.

Funding: The authors are grateful for the financial support received from the CICYT (Spain) through the research project DPI2016-80077-R.

\section{References}

[1] M. Carsí, J. Llaneza, F. Carreño, O.A. Ruano: Int. J. Mater. Res. 111 (2020) 968. DOI:10.3139/146.111965

[2] G. Lothongkum, P. Wongpanya, S. Morito, T. Furuhara, T. Maki: Corros. Sc. 48 (2006) 137. DOI:10.1016/j.corsci.2004.11.017

[3] H. Berns, A. Kühl: Wear 256 (2004) 16. DOI:10.1016/S0043-1648(03)00218-7

[4] Y. Han, D. Zou, Z. Chen, G. Fan, W. Zhang: Mater. Charact. 62 (2011) 198. DOI:10.1016/j.matchar.2010.11.013

[5] S. Patra, A. Ghosh, Vinod Kumar, D. Chakrabarti, L.K. Singhal: Mater. Sci. Eng. A 660 (2016) 61. DOI: $10.1016 /$ j.msea.2016.02.067

[6] D.N. Zou, K. Wu, Y. Han, W. Zhang, B. Cheng, G.J. Qiao: Mater. Des. 51 (2013) 975. DOI:10.1016/j.matdes.2013.04.065

[7] T. Siegmund, E. Werner, F.D. Fischer: Mech. Phys. Solids 43 (1995) 495. DOI:10.1016/0022-5096(95)00003-2

[8] Y.V.R.K. Prasad, T. Seshacharyulu: Int. Mater. Rev. 43 (1998) 243. DOI:10.1179/imr.1998.43.6.243

[9] S.V.S. Narayana Murty, B. Nageswara, B.P. Kash: Int. Mater. Rev. 45 (2000) 15. DOI:10.1179/095066000771048782

[10] A.H. Sheikhali, M. Morakkabati: Int. J. Mater. Res. 111 (2020) 1. DOI:10.3139/146.111881

[11] Y.V.R.K. Prasad, K.P. Rao, S. Sasidhar: Hot Working Guide, Materials Park, OH: ASM International (2015).

[12] E. Ghasemi, A. Zarei-Hanzaki, E. Farabi, K. Tesar, A. Jäger, M. Rezaee: J. Alloys Compd. 695 (2017) 1706. DOI:10.1016/j.jallcom.2016.10.322

[13] Yashwant Mehta, S.K. Rajput, V.V. Dabhade, G.P. Chaudhari: J. Mater. Eng. Perform. 25 (2016) 1376. DOI:10.1007/s11665-016-1992-9

[14] F. Montheillet, J.J. Jonas, K.W. Neale: Metall. Trans. A 27 (1996) 232. DOI: $10.1007 / \mathrm{BF} 02647764$

[15] S. Ghosh: Metall. Mater. Trans. A 31 (2000) 2973. DOI:10.1007/BF02830342

[16] E.S. Puchi, M.H. Staia: Metall. Trans. A 26 (1995) 2895. DOI:10.1007/BF02669647 
[17] S.V.S. Narayana Murty and B. Nageswara Rao: Mater. Sci. Lett. 17 (1998) 1203. DOI:10.1023/A:1006541710533

[18] S.V.S. Narayana Murty, B. Nageswara Rao, B.P. Kashyap: Modell. Simul. Mater. Eng. 10 (2002) 503. DOI:10.1088/0965-0393/10/5/303

[19] S.V.S. Narayana Murty, B. Nageswara Rao, B.P. Kashyap: Comput. Sci. Technol. 63 (2003) 119. DOI:10.1016/S0266-3538(02)00197-5

[20] S.V.S. Narayana Murty, B. Nageswara Rao, B.P. Kashyap: J. Mater. Process. Technol. 166 (2005) 279. DOI:10.1016/j.jmatprotec.2004.09.088

[21] S.L. Semiatin, J.J. Jonas: Formability and Workability of Metals, ASM, Metals Park, Ohio (1984).

[22] H.L. Gegel: Computer Simulation in Material Science, ASM, Metals Park, Ohio (1987).

[23] J.C. Malas: Methodology for design and control of thermo-mechanical process, Ph. D. dissertation, Ohio University (1991).

[24] S. Spigarelli, E. Cerri, P. Cavaliere, E. Evangelista: Mater. Sci. Eng. A 327 (2002) 144. DOI:10.1016/S0921-5093(01)01647-1

[25] G. Zhou, H. Ding, F. Cao, B. Zhang: J. Mater. Sci. Technol. 30 (2014) 217. DOI:10.1016/j.jmst.2013.07.008

[26] J.J. Jonas, C.M. Sellars, W.J. McG. Tegart: Metall. Rev. 14 (1969) 1. DOI:10.1179/mtlr.1969.14.1.1

[27] P. Cizek, B.P. Wynne: Mater. Sci. Engi. A 230 (1997) 88. DOI:10.1016/S0921-5093(97)00087-7

[28] A. Momeni, K. Dehghani: Mater. Sci. Eng. A 528 (2010) 1448. DOI:10.1016/j.msea.2010.11.020

[29] H.J. McQueen, S. Yue, N.D. Ryan, E. Fry: Hot working characteristics of steels in austenitic state, J. Mater. Process. Technol. 53 (1995) 293. DOI:10.1016/0924-0136(95)01987-P

[30] Y. Liu, Y. Ning, Y. Nan, H. Liang, Y. Li, Z. Zhao: J. Alloys Compd. 633 (2015) 505. DOI:10.1016/j.jallcom.2015.02.010

[31] M. Srinivasan, C. Loganathan, R. Narayanasamy, V. Senthilkumar, Q.B. Nguyen, M. Gupta: Mater. Des. 47 (2013) 449. DOI:10.1016/j.matdes.2012.11.028

[32] F. Zhang, J.L. Sun, J. Shen, X.D. Yan, J. Chen: Mater. Sci. Eng. A 613 (2014) 141. DOI:10.1016/j.msea.2014.06.085

[33] A. Iza-Mendia, I. Gutierrez: Factors affecting the hot workability of duplex stainless steels, Duplex Stainless Steels 2007, International Conference and Expo, AIM. Grado, Italy (2007).
[34] J. Payne, G. Welsh, R.J. Christ, J. Nardiello, J.M. Papazian: Int. J. Fatigue 32 (2010) 247. DOI:10.1016/j.ijfatigue.2009.06.003

[35] J.S. Jin, X.Y. Wang, H.E. Hu, J.C. Xia: Met. Mater. Int. 18 (2012) 69. DOI:10.1007/s12540-012-0009

[36] Y.J. Lan, C. Pinna: A finite element model of the heterogeneous deformation of austenite and ferrite in a duplex stainless steel under hot plane strain compression, in: J. Fan, H. Chen (Eds.), Advances in Heterogeneous Material Mechanics, Huangshan, China (2008) 312.

[37] M. Hatherly: Deformation at high strains, in: R. Gifkins (Ed.), Strength of metals and alloys, Oxford, Pergamon Press (1983) 1181. DOI:10.1016/B978-1-4832-8423-1.50173-5

(Received February 11, 2021; accepted March 28, 2021)

\section{Correspondence address}

Dr. Anas Al Omar

Departament d'Enginyeria Mecànica

EPSEM - Universitat Politècnica de Catalunya

Avenida Bases de Manresa

$61-73,08242$ Manresa

Barcelona

Spain

Tel.: +34938777266

Fax: +34 938777202

E-mail: anas.al.omar@upc.edu

\section{Bibliography}

DOI 10.1515/ijmr-2021-8242

Int. J. Mater. Res. 112 (2021) 7; page 518-526

(C) 2021 Walter de Gruyter GmbH, Berlin/Boston, Germany

ISSN $1862-5282 \cdot$ e-ISSN 2195-8556 